the relationship between the local governments and the central administration. Dr. Jacques Maquet hopes to undertake a study of the Yeke of Katanga in the near future.

\title{
Education of the African Child
}

ON the initiative of the Bureau International Catholique d'Enfance, a conference was held at Yaounde (French Cameroons) in January 1957. A questionnaire had been prepared in advance of the conference, and an analysis of the replies received, together with an account of the work of the five sections of the conference and of the conclusions arrived at, are given in C.E.P.S.I., June 1957. These include the importance of carrying out an intensive psychological and sociological study of the African child and his background; existing research centres for this purpose should be further developed and their numbers increased. More teachers are needed and particular efforts should be made to ensure that parents realize their responsibilities and play their own part in the education of their children. It is important that those in charge of education should have a sound religious background which can be communicated to the children. Stress was also laid on the need for raising the standard of living, especially in rural areas, and on the improvement of the status of women; care should be taken to educate young women to fulfil the roles of wives and mothers. The task of educating the African child is one which calls for the collaboration of all.

\section{The Press in Africa}

The Press in Africa, edited by Helen A. Kitchen, is the first volume in a series of handbooks which Ruth Sloan Associates proposes to publish. It contains a preface by Ruth Sloan and an introduction, 'A Nigerian journalist looks at African newspapers', by Abiodan Aloba, and separate chapters on each of twenty-three territories. It is hoped that the second volume, $A$ Survey of American Interests in Africa, will be ready for publication in March or April I958. The third volume will be a Survey of Educational Policies and Practices in Africa.

\section{Centre Intercontinental d'Etudes de Techniques Biologiques, Paris}

LEs correspondants du C.I.É.T.B. en Afrique sont groupés selon les subdivisions en aires subcontinentales, divisées à leur tour en régions naturelles. Celles-ci sont partagées en sections correspondant généralement à un territoire administratif (État ou pays sous tutelle). A l'intérieur du C.I.É.T.B., l'Unité Afrique coopère à ses problèmes de chaque échelon, avec d'autres aires subcontinentales intertropicales (américaine, asiatique, pacifique), et avec des organismes internationaux, nationaux ou régionaux par le canal du Secrétariat Général. Le Comité Afrique est formé des Délégués des trois aires subcontinentales, A. Dziri (Tunis), J. Pagot (Bamako), J. M. Watt (Johannesburg), assistés des délégués régionaux.

Les buts généraux de la Section Anthropologique sont de coordonner les rapports entre ses deux sous-sections (Ethnologique et Anthropobiologique) au sein du C.I.É.T.B., avec les autres sections, soit par le Secrétariat Général, soit sous forme de commissions; coordonner les rapports entre spécialistes correspondant à la même discipline intéressée; poursuivre des travaux et enquêtes scientifiques et technologiques sous couvert du Secrétariat Général avec les organismes internationaux. La sous-section ethnologique a pour but d'étudier les caractères ethniques naturels des divers peuplements humains. Elle est en rapport constant avec le Bureau Géographique pour l'étude des domaines continentaux, régionaux, etc. de chaque ethnie. La sous-section anthropobiologique a pour but d'étudier par travaux de ses membres et par enquêtes auprès des autres sections du C.I.É.T.B. (Naturaliste, Sciences médicales) la Biologie normale de l'homme et des anthropoïdes, de déterminer les biotypes non-ethniques, les règles de la climatérie humaine. 
Les principaux problèmes en cours: sous-section ethnologique: Les anthropoïdes actuels doivent-ils être considérés comme une mutation du genre humain ou être définitivement séparés de celui-ci?; dans la sous-section anthropobiologique: (I) Normalisation des biotypes; (2) Climatérie comparée des types humains (caractères ethniques et caractéristiques climatiques).

[Communicated by J. S. de Goldfiem]

\section{Ancora sul cannibalismo dei 'Niam-Niam'}

IL breve scritto che pubblicai in argomento su questa rivista (vol. xxvii, no. 2, pp. 178-86, April 1957) aveva lo scopo di chiarire che non è ormai più permesso di confondere gli Azande con i 'Niam-Niam'. Del lavoro di Renzo Carmignani - che mi forniva lo spunto per tale precisazione - avevo ammesso l'utilità, tenuto conto dei materiali che l'autore aveva avuto a disposizione; deploravo solo il fatto che egli fosse caduto nella confusione accennata, come lo dimostra lo stesso titolo del suo libro: 'Il cannibalismo degli Asandè (o Niam-Niam)'.

In questa confusione incorsero in passato tutti gli autori, compreso Mgr Lagae, e v'incorsi io stesso fino a pochi anni fa. E dunque inutile che il Carmignani, ad ostentationem, si appelli a valorosi studiosi quali Larken, de Calonne-Beaufaict, Hutereau, Czekanowski, Seligman etc. Ripeto che quanto fu scritto finora sul cannibalismo nella regione si può attribuire ai 'Niam-Niam', che erano principalmente di ceppo bantu, e non agli Azande, che sono di razza sudanica. In base ai miei ultimi studi, ho dimostrato che chiamare NiamNiam gli Azande è errato, e costituisce un'offesa per gli Azande.

R. Carmignani sostiene (in Africa, vol. xxvii, no. 4, p. 398, October 1957) di non essere caduto in tale confusione: però continua a scrivere del 'Cannibalismo degli Asandè', sofisticando su un bisticcio di termini che, se era scusabile un tempo, è oggi inammissibile. Se egli desidera scrivere ancora sull' argomento, basandosi sugli scritti altrui, è da augurarsi che tenga conto della essenziale distinzione ormai acquisita agli studi etnologici : egli eviterà così di offendere gratuitamente una grande nazione africana qual' è la nazione Zande.

[Communicated by Filiberto Giorgetti] 